\title{
Effects of a Dielectric Barrier Discharge (DBD) Treatment on Chitosan/Polyethylene Oxide Nanofibers and Their Cellular Interactions
}

\author{
Mahtab Asadian' ${ }^{1}$, Heidi Declercq ${ }^{2}$, Rino Morent ${ }^{1}$, Nathalie De Geyter ${ }^{1}$ \\ ${ }^{1}$ Department of Applied Physics, Research Unit Plasma Technology (RUPT) \\ Faculty of Engineering \& Architecture, Ghent University \\ Sint-Pietersnieuwstraat 41, B4, 9000 Ghent, Belgium \\ Mahtab.Asadian@UGent.be; Rino.Morent@UGent.be; Nathalie.DeGeyter@UGent.be \\ ${ }^{2}$ Tissue Engineering Group, Department of Basic Medical Sciences \\ Faculty of Medicine and Health Sciences, Ghent University \\ De Pintelaan 185, B3, 9000, Ghent, Belgium \\ Heidi.Declercq@UGent.be
}

\begin{abstract}
In this study, chitosan (CS)/polyethylene oxide (PEO) nanofibrous mats (Ø: $166 \pm 43 \mathrm{~nm}$ ) were prepared by electrospinning and subsequently subjected to a dielectric barrier discharge (DBD) treatment in the presence of three different gases (argon, ammonia/helium and nitrogen). Afterwards, water contact angle measurements, high-resolution X-ray photoelectron spectroscopy (XPS) and scanning electron microscopy (SEM) were used to characterize the surface properties of the CS/PEO nanofibers (NFs) before and after plasma treatment. Cell behaviour of the fibers was also evaluated by live/dead fluorescence microscopy one day and seven days after seeding of human foreskin fibroblasts (HFF) on the CS/PEO NFs. In addition, the morphology of the cells on the NFs was also visualized making use of SEM.

The obtained results revealed that the water contact angle on the CS/PEO NFs could be significantly decreased from approximately $59^{\circ}$ to $10^{\circ}, 26^{\circ}$ and $43^{\circ}$ after plasma modifications in $\mathrm{NH}_{3} / \mathrm{He}, \mathrm{N}_{2}$ and $\mathrm{Ar}$ respectively. XPS results revealed that $\mathrm{N}_{2}$ and $\mathrm{NH}_{3} / \mathrm{He}$ plasma treatments result in the incorporation of nitrogen-containing functional groups while argon plasma treatment mainly result in a partial degradation of the CS/PEO polymer chains. The DBD treatments are thus able to strongly increase the hydrophilicity of the CS/PEO nanofibrous mats. In addition, it was also observed that cellular interactions on the CS/PEO NFs were also significantly increased due to these plasma treatments. Hence, an effective plasma-induced surface modification of nanofibrous CS/PEO surfaces can significantly improve the biocompatibility of CS/PEO. The best outcome of cell proliferation was noted for the argon plasma modification, which was even higher than the positive control sample although the hydrophilicity was the lowest in this case. The observations thus suggest that not only the hydrophilicity characteristics but also the presence of distinct functional groups on plasma treated CS/PEO NFs itself might have a significant influence on the observed enhanced cellular interactions.
\end{abstract}

Keywords: Electrospinning, chitosan/PEO nanofibers, non-thermal plasma treatment, cell interactions

\section{Introduction}

The application of nanofibers in tissue engineering is increasing owing to their morphological similarity to the extracellular matrix to restore damaged tissue such as cartilage, tendon, skin and nerves [1,2]. The surface properties (i.e. wettability, surface chemistry) of these engineered scaffolds play a major role in the final scaffold success in tissue engineering applications and should thus be carefully tuned [3]. The hypothesis is that an altered surface chemistry due to the incorporation of various functional groups during a non-thermal plasma treatment could have a positive impact on cell adhesion pathways, which remain poorly characterized in literature.

Chitosan (CS) is a known natural polymer obtained by alkaline deacetylation of chitin. The molecular structure of CS which is a linear polysaccharide is made out of glucosamine and $\mathrm{N}$-acetylglucosamine. As a result, CS contains a large number of hydroxy and amino groups, two functional groups which can be grafted with desirable bioactive groups. CS, thanks to its biocompatibility, biodegradability, bioactivity adhesiveness and non-toxicity together with its specific interactions with the components of the extra cellular matrix and growth factors, appears as a relevant candidate for the preparation of nanofibrous scaffolds for tissue engineering purposes [4]. Unfortunately, electrospinning of pure CS has been investigated by several authors, all of which found that the manufacturing of pure CS nanofibers was extremely 
challenging [5,6]. Researchers have instead started to focus on electrospinning blends of CS with other compatible polymers such as for example polyvinyl alcohol (PVA) and polyethylene oxide (PEO) [5, 7, 8]. Based on previous studies, blends of CS and PEO will be electrospun in this work due to the excellent electrospinning characteristics, the capability to create ultrafine fibers and the biocompatibility of PEO. In a next step, the CS/PEO nanofibrous mats will be treated with a dielectric barrier discharge (DBD), which is one of the most popular non-thermal plasmas for surface modification [9-11]. The influence of plasma treatment on the CS/PEO surface properties will be investigated in detail by X-ray photoelectron spectroscopy (XPS), contact angle measurements and atomic force microscope (AFM). In a next step, the influence of the CS/PEO surface changes on cell behaviour will be studied by examining the biological response of fibroblasts seeded onto untreated and plasma-treated CS/PEO NFs making use of scanning electron microscopy (SEM) and fluorescence microscopy. This investigation will enable us to determine if the performed DBD treatments are able to positively affect cellular interactions on CS/PEO nanofibers.

\section{Methodology}

CS (Sigma Aldrich) with a deacetylation degree of $83.8 \%$ mixed with PEO (Sigma Aldrich) was used to prepare random nanofibers by electrospinning. An electrospinning solution of 4\% (w/v) of blended CS/PEO (3:1) was prepared using a 9:1 mixture of acetic acid (Sigma-Aldrich) and water. The obtained solution was introduced to an electrospinning machine (Nanospinner24, Inovenso, Turkey) making use of the following conditions: an applied voltage of $27 \mathrm{kV}$, a distance between the needle and the collector equal to $10 \mathrm{~cm}$ and a solution flow rate set to $0.5 \mathrm{ml} / \mathrm{h}$ at room temperature. Next, the obtained NFs were modified using a DBD reactor. The discharge was generated between two round electrodes: the upper electrode is a stainless steel mesh $(\varnothing: 50 \mathrm{~mm})$, while the lower electrode is a copper plate $(\varnothing: 48 \mathrm{~mm})$ covered with A12O3. The gap between both electrodes was maintained at $1 \mathrm{~mm}$ and the mesh electrode was connected to an AC high voltage source with a frequency of $50 \mathrm{kHz}$ while the lower electrode was grounded through a capacitor of $10 \mathrm{nF}$. After the nanofibrous materials to be treated were placed on the lower electrode, the plasma reactor was pumped down to $20 \mathrm{~Pa}$ and then filled with the discharge gas for 3 minutes at a rate of 3 standard liters per minute (slm) to reach a pressure of $90 \mathrm{kPa}$. Afterwards, the gas flow rate was decreased to $1 \mathrm{slm}$ and the pressure in the plasma reactor was lowered to $5 \mathrm{kPa}$. The plasma treatments in this work were performed at $5 \mathrm{kPa}$ in three different discharge gases (argon, nitrogen, and a $10 \%$ ammonia/90\% helium mixture) at a discharge power of $2.5,6$ and $12 \mathrm{~W}$, respectively, and with a fixed treatment time of 30 seconds. The morphology of the CS/PEO NFs before and after DBD plasma treatment was characterized by scanning electron microscopy (SEM, JSM-6010 PLUS/LV, USA) to detect possible changes in the morphology of the NFs and to measure the average fiber diameter with ImageJ software. The hydrophilicity of the nanofibrous scaffolds was also examined with a drop-shape analysis system (DSA25, Krüss GmbH, Germany) by using deionized water droplets $(2 \mu \mathrm{l})$ at room temperature. The chemical composition of the polymer surfaces was determined by XPS, which was performed in a PHI Versaprobe II device, using a non-monochromatic Al Ka-beam ( $\mathrm{h} v=1486.6 \mathrm{eV}$ ) operating at $43.5 \mathrm{~W}$. All measurements were conducted in a vacuum of at least 10-6 $\mathrm{Pa}$ and the photoelectrons were detected with a hemispherical analyser positioned at an angle of $45^{\circ}$ with respect to the normal sample surface. Survey scans were recorded with a pass energy of $187.85 \mathrm{eV}$ and the elements present on the surface were identified from these scans. The samples were measured at six locations per sample and the present elements were quantified with Multipak software using a Shirley background by applying the relative sensitivity factors supplied by the manufacturer. HFF cells were cultured on the treated and untreated NFs after exposure to UV light for 30 minutes (sterilization step) at a density of 40.000 cells $/ 1000 \mu 1$ of culture media per scaffold. The cell seeded scaffolds were incubated at $37^{\circ} \mathrm{C}$ under $5 \% \mathrm{CO} 2$ for 1 and 7 days. Tissue culture polystyrene (TCPS) was used as a positive control. Live/dead cell staining was used to evaluate cell viability, the cell seeded scaffolds were incubated in $2 \mu \mathrm{l}(1 \mathrm{mg} / \mathrm{ml})$ of calcein-AM and $2 \mu \mathrm{l}(1 \mathrm{mg} / \mathrm{ml})$ propidium iodide in $1 \mathrm{ml}$ phosphate buffer saline (PBS) and were subsequently imaged with a fluorescence microscope (Olympus, IX 81) with appropriate filters. The morphology of the HFF cells was also evaluated making use of SEM after performing a cell fixation step.

\section{Results and Discussion}

First, the electrospun CS/PEO NFs were examined making use of SEM to visualize their morphology before and after plasma treatment (Fig.1). This SEM image reveals a smooth CS/PEO fiber morphology without beads and a randomly interconnected structure by using the optimized electrospinning parameters mentioned in the methodology section. The average fiber diameter was also determined and was found to be $166 \pm 43 \mathrm{~nm}$. In a next step, the CS/PEO NFs were plasma treated in nitrogen, ammonia/helium and argon. All these plasma treatments were found to strongly improve the wettability 
of the CS/PEO NFs surfaces. The untreated NFs had a contact angle of approximately $58^{\circ}$ which considerably decreased to a value of $43^{\circ}, 26^{\circ}$ and $10^{\circ}$ for the nanofibers plasma treated in argon, nitrogen and ammonia/helium, respectively. In addition, it was also observed from the SEM images of the plasma modified samples (an example after argon plasma treatment is shown in Fig.1) that the surface morphology of the NFs was not affected by the different plasma treatments.

(a)

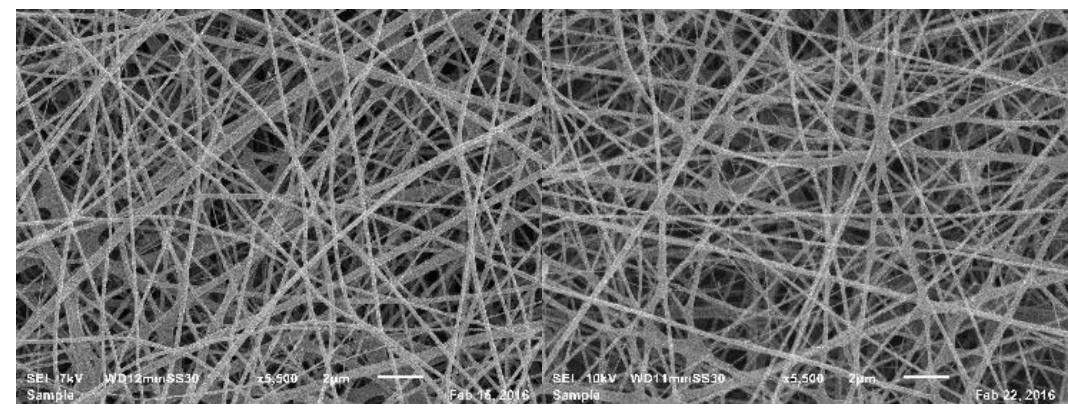

Fig. 1: SEM images of electrospun CS/PEO a) before and b) after argon plasma treatment.

In a next step, the chemical composition of the untreated and plasma treated CS/PEO NFs was examined through XPS measurements. Based on XPS survey scans, the surface elemental chemical composition was determined before and after the different plasma treatments and the results are shown in Fig. 2.

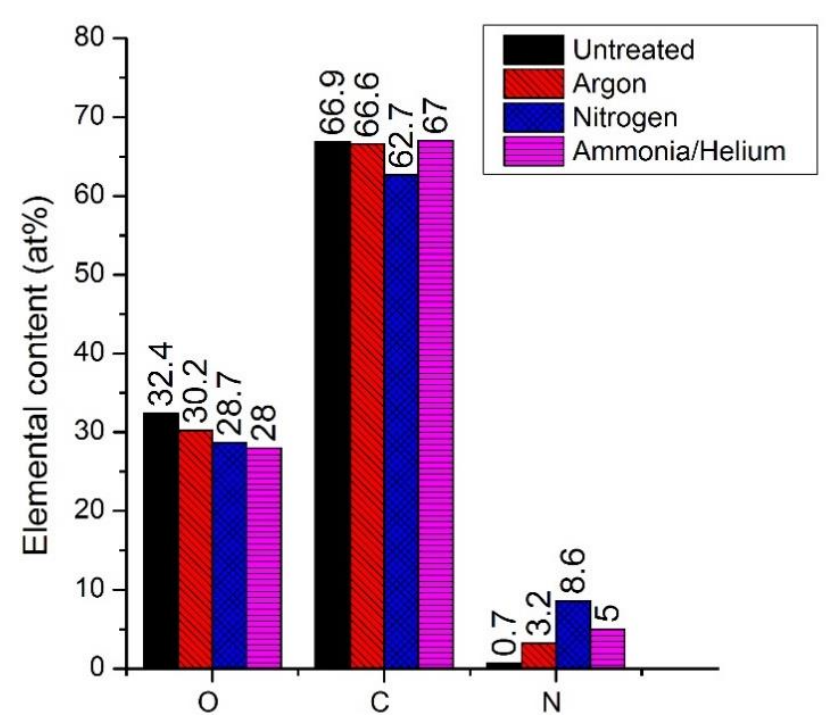

Fig. 2: Elemental composition (in at\%) of untreated and plasma treated electrospun CS/PEO NFs.

The XPS results showed that after argon, nitrogen and ammonia/helium plasma modification, there are some differences in chemical composition. The obtained results clearly suggest that the performed plasma treatments result in the incorporation of new functional groups. In case of nitrogen and helium/ $\mathrm{NH}_{3}$ plasma treatments, a significant amount of nitrogen containing groups are incorporated on the surface of the NFs as a result of the interaction of active nitrogen plasma species with the surface of the NFs. Correspondingly, the relative oxygen and carbon amounts decrease as more nitrogen atoms are added to the surface. An argon plasma treatment also seems to result into a decreasing amount of oxygen combined with an increasing amount of nitrogen, although nitrogen as such is not present in the discharge gas. One possible mechanism for this peculiar result might be the plasma-induced partial decomposition of CS/PEO molecular chains resulting into slightly different oxygen, carbon and nitrogen amounts.

The cellular interactions on the differently prepared CS/PEO scaffolds have also been investigated 1 day and 7 days after cell seeding and the images obtained by SEM and fluorescent microscopy are presented in Fig.3. These images 
clearly demonstrate strong differences in cellular interactions between the untreated sample on the one hand and the plasma treated samples on the other hand. On the untreated sample, 1 day after cell seeding, cells mostly cluster and thus poorly attach to the sample. In addition, 7 days after cell seeding, most of the cells present on the untreated sample still show clusters while also quite a few cells appear to be dead (red color in Fig.3). It can thus be concluded that cellular interactions on the untreated sample are thus rather poor. On CS/PEO samples which have been treated with the different DBDs, one can see that 1 day after cell seeding, more live cells are present on the samples while the cells also adhere better to the sample surface, which can be evidenced from their spread morphology. Comparing the results on the different plasma treated samples, one can clearly observe that the argon plasma treatment results in the best cell-material interactions as clearly shown by the more spread morphology of the cells compared to the other plasma modified samples. On all plasma treated samples, 7 days after cell seeding, a more pronounced number of HFFs is present on the NFs showing nicely elongated morphologies suggesting an excellent cell proliferation on the plasma treated NFs (as can be observed in Fig.3). In agreement with the results after 1 day, one can also again observe that the HFFs prefer the argon plasma treated sample to grow on as in this case the HFFs are almost completely covering the NFs. The better cellular interactions on the argon plasma modified samples compared to the other plasma treated samples are most likely due to the moderate surface hydrophilicity of this sample, as it is known that moderate hydrophilic surfaces can have better cell-material interactions than highly hydrophilic surfaces $[12,13]$.

(a)

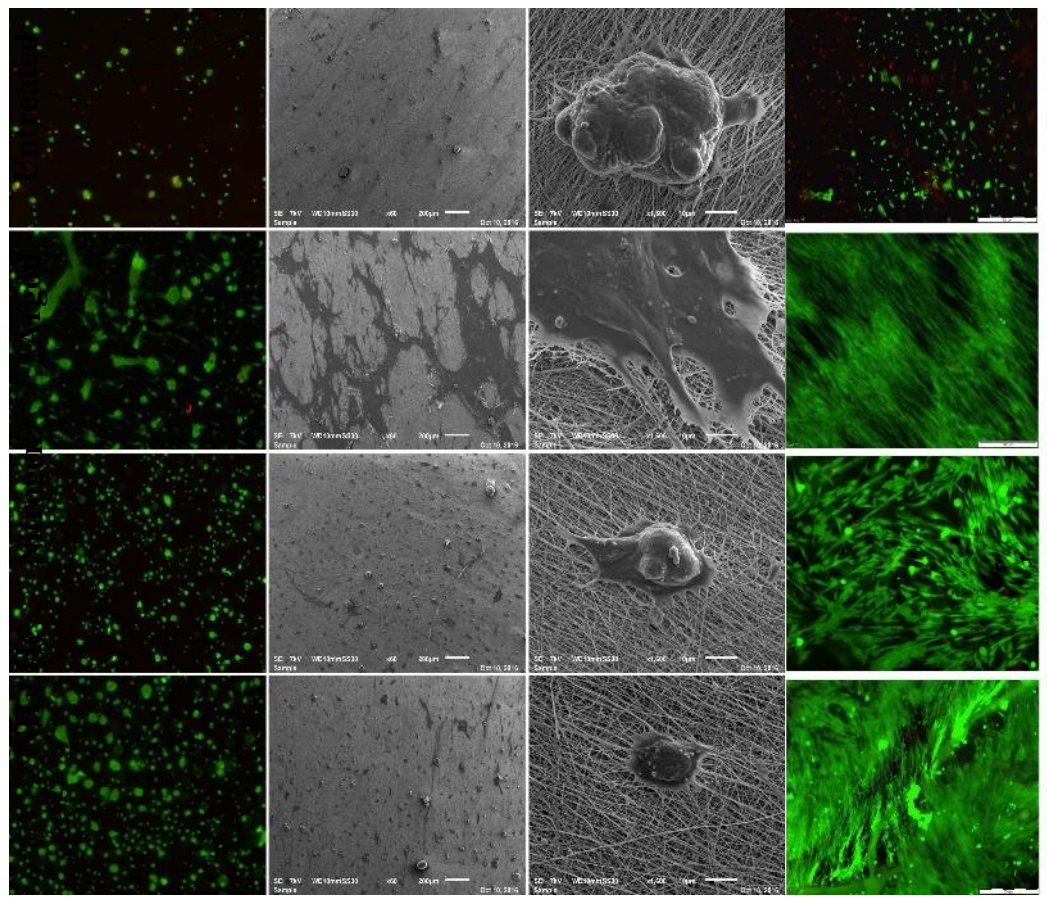

(b)

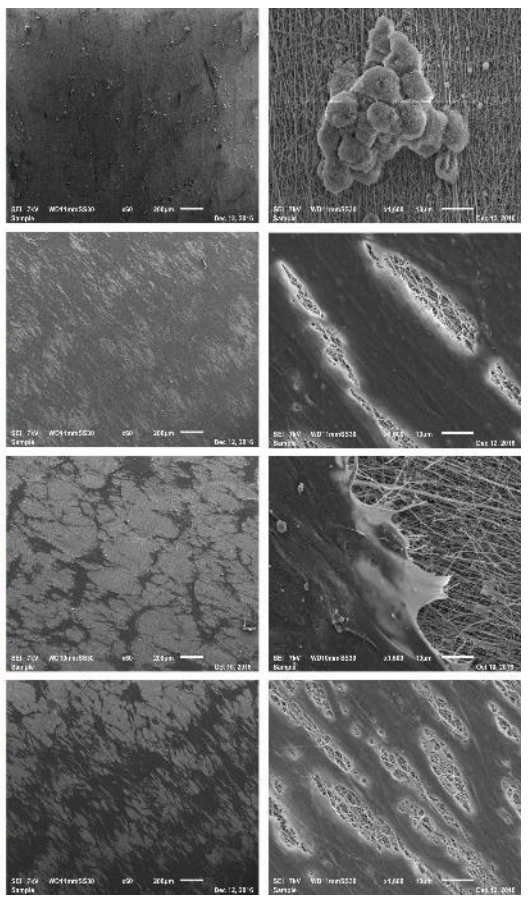

Fig. 3: Images for HFF cells at a) day 1 and b) day 7 cultured on untreated and different plasma treated CS/PEO NFs obtained by fluorescence and scanning electron microscopy.

\section{Conclusion}

In this work, non-thermal plasma treatments have been performed in different gases (argon, nitrogen and ammonia/helium) to modify the surface properties of electrospun CS/PEO NFs. It was observed that all performed plasma treatments were able to increase the hydrophilicity of the CS/PEO NFs and this without causing any morphological changes to the nanofibers. These increases in hydrophilicity could be attributed to the incorporation of nitrogen-containing functional groups after nitrogen and ammonia/helium plasma treatment and due to the plasma-induced partial degradation of CS/PEO polymer chains after argon plasma treatment. The induced chemical changes were also found to positively affect the adhesion and proliferation behavior of HFFs on the CS/PEO NFs. It was observed that more live cells were present on the plasma-modified samples and that the cells adhere and proliferate better on the plasma-modified samples which was evidenced by their spread morphology. In addition, the best cell-material interactions were observed for the 
argon plasma treated sample most likely due to the moderate hydrophilicity of this sample compared to the other plasma treated NFs, which show a very high hydrophilic character. Plasma modifications are thus able to strongly increase cellmaterials interactions on CS/PEO nanofibrous scaffolds. These results thus suggest that plasma modified NFs may have a large potential to act as tissue engineering scaffolds in the near future.

\section{Acknowledgements}

This research has received funding from the European Research Council (ERC) under the European Union's Seventh Framework Program (FP2007-2013): ERC Grant Agreement number 335929 (PLASMATS) and ERC Grant Agreement number 279022 (PLASMAPOR).

\section{References}

[1] W. J. Li, C. T. Laurencin, E. J. Caterson, R. S. Tuan, and F. K. Ko, "Electrospun nanofibrous structure: a novel scaffold for tissue engineering," Journal of biomedical materials research, vol. 60, pp. 613-621, 2002.

[2] B. Dhandayuthapani, U. M. Krishnan, and S. Sethuraman, "Fabrication and characterization of chitosan-gelatin blend nanofibers for skin tissue engineering," Journal of Biomedical Materials Research Part B: Applied Biomaterials, vol. 94, pp. 264-272, 2010.

[3] B. G. Keselowsky, D. M. Collard, and A. J. García, "Surface chemistry modulates focal adhesion composition and signaling through changes in integrin binding," Biomaterials, vol. 25, pp. 5947-5954, 2004.

[4] P. J. VandeVord, H. W. Matthew, S. P. DeSilva, L. Mayton, B. Wu, and P. H. Wooley, "Evaluation of the biocompatibility of a chitosan scaffold in mice," Journal of Biomedical Materials Research Part A, vol. 59, pp. 585590, 2002.

[5] M. Pakravan, M.-C. Heuzey, and A. Ajji, "A fundamental study of chitosan/PEO electrospinning," Polymer, vol. 52, pp. 4813-4824, 2011.

[6] K. Desai, K. Kit, J. Li, and S. Zivanovic, "Morphological and surface properties of electrospun chitosan nanofibers," Biomacromolecules, vol. 9, pp. 1000-1006, 2008.

[7] B. Duan, C. Dong, X. Yuan, and K. Yao, "Electrospinning of chitosan solutions in acetic acid with poly (ethylene oxide)," Journal of Biomaterials Science, Polymer Edition, vol. 15, pp. 797-811, 2004.

[8] L. Li and Y.-L. Hsieh, "Chitosan bicomponent nanofibers and nanoporous fibers," Carbohydrate research, vol. 341, pp. 374-381, 2006.

[9] T. Desmet, R. Morent, N. D. Geyter, C. Leys, E. Schacht, and P. Dubruel, "Nonthermal plasma technology as a versatile strategy for polymeric biomaterials surface modification: a review," Biomacromolecules, vol. 10, pp. 2351$2378,2009$.

[10] R. Morent, N. De Geyter, T. Desmet, P. Dubruel, and C. Leys, "Plasma surface modification of biodegradable polymers: a review," Plasma Processes and Polymers, vol. 8, pp. 171-190, 2011.

[11] N. De Geyter, R. Morent, and C. Leys, "Surface modification of a polyester non-woven with a dielectric barrier discharge in air at medium pressure," Surface and Coatings Technology, vol. 201, pp. 2460-2466, 2006.

[12] J. H. Lee, G. Khang, J. W. Lee, and H. B. Lee, "Interaction of different types of cells on polymer surfaces with wettability gradient," Journal of colloid and interface science, vol. 205, pp. 323-330, 1998.

[13] J. H. Lee, J. W. Lee, G. Khang, and H. B. Lee, "Interaction of cells on chargeable functional group gradient surfaces," Biomaterials, vol. 18, pp. 351-358, 1997. 